\title{
1 Range size heritability and diversification patterns in 2 the liverwort genus Radula
}

4 Jairo Patiño $^{\text {a,b,c, } * \text {, Jian Wang }}{ }^{\text {d }}$, Matt A.M. Renner ${ }^{\text {e }}$, S. Robbert Gradstein ${ }^{\mathrm{f}}$, Benjamin

5 Laenen $^{\mathrm{g}}$, Nicolas Devos ${ }^{\mathrm{h}}$, A. Jonathan Shaw ${ }^{\mathrm{h}}$, Alain Vanderpoorten ${ }^{\mathrm{b}, \mathrm{c}}$

6

$7 \quad$ a Island Ecology and Evolution Research Group, Instituto de Productos Naturales y

8 Agrobiología (IPNA-CSIC), Astrofísico Francisco Sánchez 3, La Laguna, Tenerife,

9 Canary Islands, 38206, Spain

$10{ }^{\mathrm{b}}$ Department of Biology, Ecology and Evolution, Institute of Botany, University of Liège,

11 Liège, Belgium

$12{ }^{\mathrm{c}}$ Azorean Biodiversity Group (GBA, CITA-A) and Platform for Enhancing Ecological

13 Research \& Sustainability (PEERS), Universidade dos Açores, Angra do Heroísmo,

14 Terceira, Açores, Portugal

$15{ }^{\mathrm{d}}$ Bryology Laboratory, School of Life Sciences, East China Normal University, Shanghai

16 200241, China

17 e National Herbarium of New South Wales, Royal Botanic Gardens Sydney, Mrs

18 Macquaries Road, Sydney, NSW 2000, Australia

19 f Département de Systématique et Evolution, Museum National d' Histoire Naturelle,

20 Paris, France

$21 \mathrm{~g}$ ScienceLifeLab, Stockholm University, Solna, Sweden

$22{ }^{\mathrm{h}}$ Department of Biology, Duke University, Durham, NC, USA.

23

$24 *$ Corresponding author. Address: Island Ecology and Evolution Research Group,

25 Instituto de Productos Naturales y Agrobiología (IPNA-CSIC), La Laguna, Tenerife,

26 Canary Islands, 38206, Spain. Email address: jpatino.llorente@gmail.com

27 


\section{ABSTRACT}

29 Why some species exhibit larger geographical ranges than others, and to what extent does

30 variation in range size affect diversification rates, remains a fundamental, but largely

31 unanswered question in ecology and evolution. Here, we implement phylogenetic

32 comparative analyses and ancestral area estimations in Radula, a liverwort genus of

33 Cretaceous origin, to investigate the mechanisms that explain differences in geographical

34 range size and diversification rates among lineages. Range size was phylogenetically

35 constrained in the two sub-genera characterized by their almost complete Australasian

36 and Neotropical endemicity, respectively. The congruence between the divergence time

37 of these lineages and continental split suggests that plate tectonics could have played a

38 major role in their present distribution, suggesting that a strong imprint of vicariance can

39 still be found in extant distribution patterns in these highly mobile organisms.

40 Amentuloradula, Volutoradula and Metaradula species did not appear to exhibit losses of

41 dispersal capacities in terms of dispersal life-history traits, but evidence for significant

42 phylogenetic signal in macroecological niche traits suggests that niche conservatism

43 accounts for their restricted geographic ranges. Despite their greatly restricted

44 distribution to Australasia and Neotropics respectively, Amentuloradula and

45 Volutoradula did not exhibit significantly lower diversification rates than more

46 widespread lineages, in contrast with the hypothesis that the probability of speciation

47 increases with range size by promoting geographic isolation and increasing the rate at

48 which novel habitats are encountered. We suggest that that stochastic long-distance

49 dispersal events may balance allele frequencies across large spatial scales, leading to low

50 genetic structure among geographically distant areas or even continents, ultimately

51 decreasing the speciation rates in highly mobile, widespread lineages. 


\section{1. Introduction}

54 Geographical ranges tremendously vary among species (Lester et al., 2007; Abellán and

55 Ribera, 2011; Laube et al., 2013a, b), but in fact, also vary during the lifespan of a

56 species depending on intrinsic (the proportion of the ancestor's range inheritance) and

57 extrinsic (geological and climatic history) factors until decline and extinction (Jones et

58 al., 2005; Sheth et al., 2014; Fine, 2015). Four main models of range size transformations

59 have been proposed (Gaston, 1996, 1998). In the 'age and area' and 'stasis' models,

60 species lifespans and range sizes are correlated, but the two models differ by the cause-

61 and-effect relationship between these two factors: species with long lifespans are

62 expected to exhibit larger ranges than short-lived species in the former, whereas species

63 with large range sizes are expected to have longer lifespans than species with small

64 ranges in the latter. Under the 'random' model', conversely, range sizes continuously

65 vary depending on environmental factors. In the 'cyclical model', there is a rapid range

66 size increase post-speciation, followed by a steady decline to extinction.

67 If, as the 'stasis model' implies, geographic range determines the probability of

68 persistence of lineages, we expect that, following Jablonski's hypothesis (Jablonski,

69 1987, but see Webb and Gaston, 2003) based on geographic range reconstructions of

70 Cretaceous mollusks, range size is under selection and is heritable. Furthermore, under

71 the 'age and area model', we expect to observe a trend towards progressive increases of

72 range size with lineage age. Trait heritability over a phylogeny can be measured through

73 several estimators, such as Pagel's lambda (Pagel, 1999b), which reveals whether the tree

74 correctly predicts the character states observed in present taxa. If range size globally

75 tends to increase or decrease as the 'age and area' models suggest, one would further 
76 expect that a directional random-walk model, which includes a parameter measuring the

77 regression of trait values across species against total path length from the root of the tree

78 to the tips (Pagel, 1999a), describes the data significantly better than a constant-variance

79 random walk model.

80 Measures of phylogenetic signal are, however, based on the distribution of traits observed

81 in species and then 'evolved' along the phylogeny, assuming that the relationship

82 between the traits of ancestors and descendants can be inferred from the distribution of

83 traits among descendants. This may, however, be a problematic assumption in the context

84 of geographic range-size heritability (e.g. Gaston, 1998; Hunt et al., 2005). In fact,

85 species-level heritability of range sizes may result from two mechanisms, namely

86 phylogenetic conservatism of ecological traits such as species niche (Sheth et al., 2014),

87 dispersal ability (Lester et al., 2007) and mating systems (Randle et al., 2009), and direct

88 inheritance of ranges (vicariance) (Borregaard et al., 2012). In the first case, the sharing

89 of traits that are strong determinants of range size is expected to lead closely related

90 species to exhibit more similar range sizes than distantly related ones, indeed resulting in

91 significant lambda statistics. In the second case, conversely, the asymmetric division of

92 an ancestral range (vicariance) will tend to make the range sizes of sister species more

93 dissimilar (Waldron, 2007). As a result, direct range inheritance cannot be detected using

94 lambda estimators.

95 Finally, if range size is indeed heritable and controls for lineage persistence through time,

96 we expect that range size also impacts on diversification patterns (Jablonsky and Roy,

97 2003). Under Jablonski's hypothesis (Jablonski, 1987), widely distributed species,

98 persisting for longer, are expected to exhibit lower extinction rates. In turn, larger areas 
99 are expected to provide greater opportunities for genetic isolation due to a higher

100 incidence of dispersal barriers and/or greater habitat heterogeneity for ecological

101 speciation (Kisel and Barraclough, 2010; Losos and Alder, 1996; Rabosky and Lovette,

102 2008; Rosenzweig, 1995). Following long-distance dispersal, similarly, either founder

103 effects or exposure to new habitats and selective pressures can trigger speciation (Wessel

104 et al., 2013). Conversely, the combination of geographical boundaries limiting clade

105 distributions and restricted availability of ecological opportunities is expected to lead to a

106 decrease in diversification rates. Therefore, if, as theory predicts (Goldberg et al., 2011;

107 Wagner and Erwin, 1995), the probabilities of speciation are dependent on the

108 geographical extent of the species, variations in the rates of diversification over time and

109 among lineages are expected (Losos and Alder, 1996).

110 In the present study, we focused on Radula, a leafy liverwort genus that, with its

111 Cretaceous origin (Cooper et al., 2012; Laenen et al., 2014) and ca. 200 species with

112 diverse geographic ranges, appears as an ideal model to investigate the evolution of range

113 sizes through time and their impact of diversification patterns. Indeed, earlier phylogenies

114 for the genus (Devos et al., 2011a; Devos et al., 2011b; Patiño et al., 2013b; Renner et al.,

115 2014; Renner et al., 2013) resolved a mixture of nearly cosmopolitan lineages (subgenera

116 Radula and Cladoradula) and more geographically restricted lineages (Amentuloradula

117 and Odontoradula, with a concentration of species in Australia and New Zealand,

118 Metaradula in the paleotropics, and Volutoradula in Central and South America). More

119 precisely, we addressed the following questions: (i) Is range size phylogenetically

120 constrained? If so, is there a trend across the tree so that geographic ranges progressively

121 become larger or smaller with the taxon age? (ii) What are the mechanisms accounting 
122 for range size heritability? In particular, are macroecological niche traits also

123 phylogenetically constrained, and/or does heritability correlate with variation in dispersal

124 life-history traits? And (iii) to what extent does variation in geographic range correlate

125 with shifts in diversification rates?

\section{2. Materials and methods}

\section{2.1. Taxon sampling and molecular protocols}

129 Building on previous work focusing on the infra-generic classification of Radula (Devos

130 et al., 2011a; Devos et al., 2011b; Patiño et al., 2013b; Renner et al., 2013), we sequenced

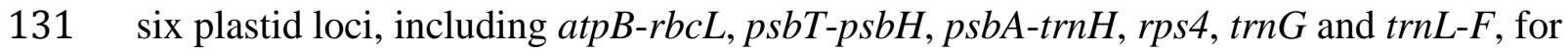

132 another 25 species (see Devos et al., 2011a, for details on the molecular protocols). With

133108 species, our sampling includes $c a .50 \%$ of the currently recognized species in the

134 genus (Devos et al., 2011b; Yamada, 2003). Devos et al. (2011b) proposed an

135 infrageneric classification for Radula with a total of seven subgenera. Two of these

136 subgenera, Amentuloradula (which encompasses $c a .16 \%$ of the total species diversity in

137 Radula) and Odontoradula (ca. $12 \%$ ), are mostly restricted to Australasia, Volutoradula

138 (ca. $23 \%)$ is mostly restricted to Central and South America, Metaradula (ca. 17\%) is

139 Pantropical but largely restricted to the Paleotropics, and both Radula (ca. $26 \%$ ) and

140 Cladoradula (ca. $6 \%$ ) are nearly cosmopolitan. The last subgenus, Dactyloradula,

141 includes a single species, the northern amphi-Pacific disjunct $R$. brunnea Steph.

142 Diplophyllum obtusifolium, Frullania moniliata, Jubulopsis novaezelandiae,

143 Jungermannia atrovirens, Lejeunea tuberculosa, Lepidolaena clavigera, Mylia taylorii

144 and Porella navicularis were employed as outgroups based on Laenen et al. (2014). 
145 Herbarium voucher numbers and GenBank accession numbers are provided in the

146 Supporting Information (Tables S1 and S2).

\section{$148 \quad$ 2.2. Character scoring}

149 The range size of each species was described by its number of occurrences among the

150 twenty world floristic regions defined by van der Wijk et al. (1959) with subsequent

151 refinements by Tan and Pócs (2000). Dispersal life-history traits (Table S3) included both

152 variation in sexual condition (monoecious vs dioecious), which controls spore

153 production, and the ability to produce specialized asexual diaspores, which were recently

154 shown to complement sexual spores in enhancing the long-distance dispersal capacities

155 of bryophytes (Laenen et al., 2016). Elevation, substrate, and biome ranges were used to

156 describe the amplitude of the macro- and microecological niche for each Radula species

157 (Table S3). To determine the elevation range, we considered five categories, which were

158 defined according to the breadth of the altitudinal range that Radula species occupy, as

159 follows: (1) very narrow range [<500 m]; (2) narrow range [< $1000 \mathrm{~m}]$; (3) medium

160 range $[<2000 \mathrm{~m}]$; (4) large range $[<3000 \mathrm{~m}]$; and very large range [> $3000 \mathrm{~m}] . \mathrm{We}$

161 defined substrate range as the number of substrates (as a proxy of microecological

162 conditions) where the species occurs. We considered: (1) rocks; (2) barks including

163 trunks and canopies; (3) leaves; and (4) soils. Biome range was based on the number of

164 biomes (defined according to Olson et al., 2001) occupied by each species, which were

165 grouped as follows: (1) Tundra; (2) Boreal forest and Taiga; (2) Montane grasslands and

166 shrublands; (3) Temperate coniferous forests and Temperate broadleaf and mixed forests;

167 (4) Tropical and sub-tropical dry, coniferous and moist broadleaf forests; (5) 
168 Mediterranean forests, woodlands and scrublands; (6) Tropical and sub-tropical

169 grasslands, savannas and shrublands; (7) Temperate grasslands, savannas and shrublands;

170 and (8) Flooded grasslands and savannas. We gathered the dataset for these ecological

171 attributes from the sources listed in Table S3.

172

173 2.3. Phylogenetic and molecular dating analyses

174 The phylogeny was reconstructed using BEAST 1.8.0 (Drummond et al., 2012). The 175 best-fitting substitution model for each individual locus was selected with the Akaike 176 information criterion using MrModelTest (Nylander, 2004) in conjunction with PAUP*

177 (Swofford, 2002). A General Time-Reversible (GTR) model with gamma-distributed rate

178 variation across sites and a proportion of invariable sites was selected for $p s b A$-trnH,

$179 r p s 4, \operatorname{trn} L-F, a t p B-r b c L$, and $\operatorname{trn} G$, while a Hasegawa, Kishino and Yano (HKY) model

180 with gamma distributed rate variation across sites and a proportion of invariable sites was

181 selected for $p s b T-p s b H$. Since the phylogeny was used to perform diversification analyses

182 and comparative analyses of trait evolution, and given that trait evolution is related to

183 time, but not necessarily to the number of substitutions (Cusimano and Renner, 2014;

184 Pellicer et al., 2014), trees were ultrametricized. Using the Akaike information criterion

185 through Markov chain Monte Carlo (AICM; Baele et al., 2012) with 1000 bootstraps in

186 BEAST, a strict molecular clock was rejected (AICM of 58930.8 and 58557.8 for the

187 strict vs. lognormal relaxed, respectively) and, therefore, an uncorrelated lognormal

188 relaxed molecular clock was employed for the subsequent dating analysis. Although two

189 Baltic amber fossils of Radula have been described, their taxonomic position within the

190 genus is uncertain, so that they represent a minimum constraint for the crown node 
191 (Feldberg et al., 2014). Therefore, to facilitate the interpretation of the trees, we

192 transformed branch lengths into actual time periods by using absolute nucleotide

193 substitution rate sampled from a log-uniform prior distribution according to the results of

194 relaxed-clock analyses across the Liverwort Tree of Life (Laenen et al., 2014), with a

195 mean of 5.273E-4 and SD of 6.463E-6 substitutions/site/million of year. A Yule prior on

196 branching rates was employed and four independent chains of $150,000,000$ generations

197 were sampled every 10,000 generations. Convergence and mixing of the sampled

198 parameters were checked using Tracer 1.6 (Rambaut et al., 2014). After discarding the

199 burn-in steps (10\%), runs were combined to obtain an estimate of the posterior

200 probability distributions for the dates of divergence.

\subsection{Ancestral area estimation}

203 To visualize the evolution of range size across a subset of 100 BEAST trees randomly 204 sampled from the posterior probability distribution obtained from BEAST to account for 205 phylogenetic uncertainty, we used the package BioGeoBEARS (Biogeography with 206 Bayesian Evolutionary Analysis in R Scripts; Matzke, 2014). BioGeoBEARS calculates

207 maximum likelihood estimates of the ancestral states at internal nodes by modeling 208 transitions between geographical ranges along phylogenetic branches as a function of 209 time. It allows the use of the Lagrange DEC model (Dispersal Extinction Cladogenesis) 210 and a new model called BioGeoBEARS DEC + J model (Matzke, 2014 and references

211 therein). Both models include two free parameters $(\mathrm{d}=$ dispersal and $\mathrm{e}=$ extinction $)$, but

$212 \mathrm{DEC}+\mathrm{J}$ includes the additional parameter $\mathrm{j}$ that corresponds to founder event speciation 
213 (Matzke, 2013). Likelihood values of these models were compared using Likelihood 214 Ratio Test.

215 Since different ancestral area reconstructions are based on different assumptions

216 and can produce conflicting results (Matzke, 2013, 2014; Pirie et al., 2012), we compared

217 these two versions of the DEC model with a likelihood version of the Dispersal-

218 Vicariance Analysis (DIVALIKE), and a likelihood version of the range evolution model

219 of the Bayesian Binary Model (BAYAREA) of RASP (Yu et al., 2015). In

220 BioGeoBEARS, founder-event speciation can be added to any of these previously

221 described models, and its relative probability can be left as a free parameter, which is

222 estimated from the data, also creating "DIVALIKE+J" and "BAYAREA+J" models. We

223 used Akaike Information Criterion (AIC) to directly compare how well the different

224 biogeography models fit the data and the best model was represented on the maximum

225 clade credibility (MCC) tree.

226 For the reconstruction of ancestral range areas, we considered eight world

227 geographical areas by merging some of van der Wijk et al.'s original regions, following

228 criteria provided by Sanmartín and Ronquist (2004), but with a few modifications from

229 Chacón and Renner (2014) to accommodate the Northern Hemisphere regions. For the

230 definition of these eight geographical areas, we also considered the evidence available for

231 floristic relationships in liverworts (Vanderpoorten et al., 2010) and the distribution of

232 strongly restricted Radula subgenera (Devos et al., 2011b). Specifically, we scored

233 occurrences in: Asia, including western Indonesia (S); sub-Saharan Africa (F); North

234 America (N); South and Central America including Mexico and southern California (A);

235 European, Mediterranean and Irano-Turanian regions, including Europe, northern Africa, 
236 Anatolia, and parts of Jordan (E); New Zealand (Z); Australia, including New Guinea

237 (T); and Pacific archipelagos (O) (Table S4).

240 Phylogenetic signal in for geographic range size, elevational range, substrate range, and

241 biome range, was quantified in terms of Pagel's $\lambda$ (Pagel, 1999b) and Blomberg's K

242 (Blomberg et al., 2003) using ML estimators implemented in the R package phytools

243 (Revell, 2012) on the 100 randomly sampled BEAST trees. To obtain statistical

244 significance ( $P$ value) for Blomberg's K, we randomly shuffled the character states

245 among taxa 100 times. Because the calculation of Pagel's

$246 \lambda \begin{array}{ll} \\ 24\end{array}$

247 et al. (2012) in contrasting the fit of a model with $\lambda \square \square$ (no phylogenetic signal) to a

248 model where $\lambda$ is not constrained. Significance of the difference of fit between the two

249 models was assessed by a likelihood ratio test (LRT). The phylogenetic and non-

250 phylogenetic models include the same number of parameters, and thus their likelihood

251 values were directly comparable, with a difference of 2.0 being considered significant

252 (Mooers and Schluter, 1999). However, the presence of phylogenetic signal can vary

253 depending on the taxonomic level because antagonistic patterns at different levels can

254 obscure one another when the analysis of phylogenetic signal is conducted

255 simultaneously across the entire phylogeny (Machac et al., 2011). The analyses were

256 therefore run individually for each subgenus (except for Dactyloradula and Cladoradula

257 due to their small size) across the 100 randomly sampled BEAST trees to account for

258 phylogenetic uncertainty and the results were summarized on the MCC tree. 
We further determined, if variation in geographic range is phylogenetically

260 constrained, whether it varies with age by comparing the fit of two models to the data, as

261 implemented by the Continuous function of BAYESTRAITS 2.0 (Pagel et al., 2004).

262 Model A is a constant-variance random-walk model, whereas model B is a directional

263 random-walk model measuring the regression of trait values (here, range size) across

264 species against total path length (Pagel, 1999b). These two models were fitted by ML

265 onto the 100 randomly sampled trees and their goodness-of-fit compared with a

266 likelihood ratio test.

267

268 2.6. Phylogenetic correlation between range size and dispersal traits

269 The correlation between range size and dispersal life-history traits was investigated by

270 comparing the fit of two nested models to the observed data as implemented by the

271 Continuous function of BAYESTRAITS. In the independent model, trait covariance was

272 set to 0 , while in the dependent model, covariance was unconstrained. We used a LRT to

273 determine whether the dependent model returned a significantly higher log-likelihood

274 than the independent model on each of the 100 trees sampled from the posterior

275 probability distribution of the BEAST analysis. Although Continuous is primarily

276 designed for the analyses of continuous characters, correlations between continuous and

277 discrete variables are computed using the same formulas as correlations between

278 continuous variables. A justification for the use of the same statistics for mixtures of

279 binary and continuous variables is that, according to Wright (1934) threshold model for

280 discrete traits, an unobserved quantitative character, termed the liability, determines the

281 state of discrete characters based on whether the liability exceeds a threshold value. 
282 Under the threshold model, underlying liabilities of discrete traits and continuous

283 characters are assumed to evolve through covarying Brownian motion, allowing

284 correlation between discrete and continuous characters (Lambert and Wiens, 2013).

\subsection{Diversification analyses}

287 We used BAMM 1.0 (Bayesian Analysis of Macroevolutionary Mixtures; Rabosky, 288 2014; Rabosky et al., 2014b) to look for shifts in speciation and net diversification rates

289 (speciation minus extinction) in Radula based on the MCC tree produced by BEAST. The

290 general model assumes that phylogenetic trees have been shaped by a heterogeneous

291 mixture of distinct evolutionary regimes of speciation and extinction. We assumed that

292 the extinction rate is constant through time within regimes. However, we avoid

293 interpreting extinction rates because of substantial controversy over the meaning of this

294 parameter as estimated from molecular phylogenies (e.g., Quental and Marshall, 2009,

295 2010; Rabosky et al., 2014a; Rabosky et al., 2014b). We accounted for incomplete taxon

296 sampling using the analytical correction, as implemented in BAMM (Rabosky, 2014;

297 Rabosky et al., 2013). We estimated that our sampling included between 30\% and 100\%

298 of the species diversity depending on the subgenus (Table S5). All visualization was

299 performed using R and C++ codes available at www.bamm-project.org (Rabosky et al., 300 2014b).

301 To summarize diversification histories and avoid some of the challenges

302 associated with interpreting all distinct shift configurations (Rabosky et al., 2014a), as

303 well as to display marginal probabilities for all rate shifts, we used macroevolutionary

304 cohort analysis, which depicts the pairwise probability that any two lineages or clades 
305 share a common set of macroevolutionary rate parameters. For a given sample from the

306 posterior distribution simulated with BAMM, a pair of species is assigned a value of 1 if

307 they inherit a common rate dynamic and a value of 0 if they have different rate dynamics.

308 A mean value is then computed over the full set of sampled shift configurations in the

309 posterior distribution. A value of 0.5 implies that $50 \%$ of the samples in the posterior

310 distribution include the focal species within a common macroevolutionary rate regime.

\section{3. Results}

313 In the MCC chronogram, all subgenera were recovered as monophyletic with high

314 support (pp from 0.99 to 1.0 ; Fig. 1). The crown age of the genus was dated in the late

315 Jurassic, with the majority of extant species originating between the Cretaceous and

316 Eocene (Table 1).

$317 \quad$ Range size was not phylogenetically constrained at the root node but exhibited 318 significant Pagel's $\lambda$ values on $51.7 \%$ of the 100 BEAST trees in Metaradula and on

$319100 \%$ of the trees in Amentuloradula and Volutoradula, respectively (Table 2; Fig. 2).

320 Blomberg's K values showed a similar pattern (Tables 2 and S6; Fig. S1).

321 In the ancestral area estimations, the DEC+J model was identified as the best-

322 fitting model (Table 3). There was an overall high uncertainty in ancestral area

323 estimations (Fig. 3), but the pattern of range size heritability described above precisely

324 corresponds to subgenera that were reconstructed to be of Australasian origin, with a

325 probability of 0.29 in Metaradula and 0.80 in Amentuloradula, and of Neotropical origin

326 with a probability of 0.81 in Volutoradula (Table 1, Fig. 3). 
328 Pagel's $\lambda$ values at the root node (Tables 2 and S6). Elevation range was significantly

329 constrained in Amentuloradula, wherein species exhibited the lowest range as compared

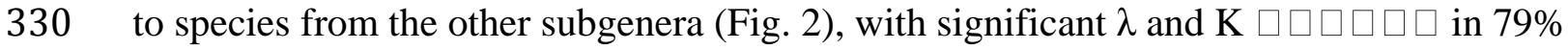

331 and $19.5 \%$ of the 100 sampled trees, respectively (Tables 2 and S6, Figs. 2 and S1).

332 Biome range was significantly constrained in Odontoradula, with significant $\lambda$ and $\mathrm{K}$

333 values also observed in 69\% and 1\% of the 100 sampled trees, in Volutoradula with

334 significant $\lambda$ and $\mathrm{K}$ values on the in $61 \%$ and $88 \%$, and in Metaradula with significant $\lambda$

335 and $\mathrm{K}$ in $33 \%$ and 55\%, respectively (Tables 2 and S6, Figs. 2 and S1). Substrate range

336 showed significant $\lambda$ and $\mathrm{K}$ values for a marginal number of the 100 sampled trees (see

337 Tables 2 and S6).

338 The LRT for directional trends showed that the log-likelihood values of Model A

339 (constant-variance random-walk model) mostly tended to be equally large or even

340 significantly larger (in 11\%) than those of Model B (directional random-walk model),

341 rejecting the directional model of geographic range size evolution in $100 \%$ of the trees

342 tested. Range size did not correlate with mating system and production of asexual

343 diaspores in any of the trees evaluated $\left(\chi^{2}\right.$ distribution with one degree of freedom; $P>$

$3440.05)$.

345 We found strong evidence for a shift in speciation rates in Radula (posterior

346 probability of a single rate model $=0.06$; posterior probability density of one rate shift $=$

347 0.91). We estimated the $95 \%$ credible set of rate shift configurations, for which just these

348 two configurations accounted for approximately 99\% of the posterior (Table A7 and Fig.

349 S2), in the form of acceleration in the rate of net diversification for subgenus Radula (see 
350 diversification-through-time trajectories in Fig. 4 and Fig. S2). In the macroevolutionary

351 cohort matrices of BAMM (Fig. 5), the color of each individual cell depicts the average

352 correlation in rate regimes between two species averaged across the MCC tree. This plot

353 consistently revealed a cohort of four distinct lineages that are governed by common sets

354 of macroevolutionary rate parameters, including Cladoradula, Dactyloradula, Radula

355 and for the rest of subgenera, respectively.

357 4. Discussion

358 Range size was phylogenetically constrained in Amentuloradula and Volutoradula, the

359 two subgenera characterized by their almost complete Australasian and Neotropical

360 endemicity, respectively, and in Metaradula, greatly restricted to Paleotropics. The

361 timing of diversification of those clades was difficult to assess in the absence of reliable

362 fossil records within Radula (Feldberg et al., 2014), leading us to implement a secondary

363 calibration. The accuracy of secondary calibrations has been an area of concern, some

364 studies showing high levels of congruence between the dates obtained from primary and

365 secondary calibrations (Aigoin et al., 2009), whereas others showed otherwise (Sauquet

366 et al., 2012). The fact that we obtained a crown age for the genus Radula (144.6 Ma,

367 109.6-197.7 HPD; Table 2) that is compatible with that found in other studies using fossil

368 calibrations [e.g., Cooper et al. (2012) (132 Ma, 79.8-182 HPD) and Feldberg et al.

369 (2014), with a mean age of $119.5 \mathrm{Ma}$, suggests that the time estimates obtained in the

370 present study are robust. Furthermore, molecular dating in general (Wilf and Escapa,

371 2015), and the use of secondary calibrations in particular (Sauquet et al., 2012), tend to

372 underestimate the actual age of lineages, reinforcing our vicariantist interpretation of the 
373 divergences of some of the oldest subgenera in the genus Radula. Thus, subgenera

374 Amentuloradula and Radula diverged from each other during a period of time

375 encompassing the Early Paleocene and the Early Cretaceous (62-107 Ma), in both cases

376 slightly before or coincident with the drift of Zealandia (i.e., New Zealand and New

377 Caledonia) from the supercontinent Gondwana. Similarly, the separation of Metaradula

378 from Volutoradula occurred 47-85 Ma, in coincidence with the separation of Australia

379 from Antarctica during the Late Cretaceous (Woodburne and Case, 1996). The high

380 degree of historical association of these two clades within a single Gondwanan plate and

381 divergence times that are contemporary with the break-up of these plates might suggest

382 that plate tectonics could have played a major role in their present distribution. As

383 Vanderpoorten et al. (2010) hypothesized based on analyses of worldwide distribution

384 patterns in liverworts, our results therefore suggest that a strong imprint of vicariance can

385 still be found in extant distribution patterns in these highly mobile organisms. Signatures

386 of ancient vicariance have been demonstrated in amphibians (Pyron, 2014), birds

387 (Ericson et al., 2002), and mammals (Hedges et al., 1996) but were erased in extant

388 distribution patterns of many higher plants (but see Mao et al., 2012; Sanmartín and

389 Ronquist, 2004) and were, indeed, unexpected in mobile spore-producing taxa like

390 bryophytes and pteridophytes (Lewis et al., 2014; Sun et al., 2014; Wolf et al., 2001),

391 wherein evidence for vicariance is limited (Korall and Pryer, 2014).

392 Although some phylogenetic constraint was present in the evolution of range size

393 in the genus Radula, no significant tendency for larger or smaller ranges with age was

394 observed. In fact, although the subgenera Radula, Volutoradula and Metaradula share

395 similar crown ages, their extant distribution is almost cosmopolitan in the former, 
396 pantropical in the latter, and Neotropical in Volutoradula. In bryophytes globally, some

397 species exhibit very old stem ages but are currently geographically very restricted, such

398 as Alophosia azorica in Azores and Hedenasiatrum percurrens in Madeira, whose stem

399 age of 100 and 40 and Ma unambiguously point to a palaeoendemic origin

400 (Vanderpoorten et al., 2011), whereas others, such as Bryoxophium norvegicum, with a

401 stem age of $100 \mathrm{Ma}$, are widely distributed, although not necessarily common, across

402 continents (Patiño et al., 2016).

403 The mechanisms explaining such differences of range size in bryophytes globally,

404 and in Radula in particular, with the largely endemic in-situ diversification of

405 Amentuloradula and Volutoradula species and their almost complete failure to disperse

406 elsewhere, despite their ancient origin, are difficult to assess. Amentuloradula and

407 Volutoradula species did not appear to exhibit losses of dispersal capacities in terms of

408 dispersal life-history traits. In fact, in contrast to a previous meta-analysis of life-history

409 traits of island bryophytes that demonstrated a significant decrease in sporophyte

410 production and increase in clonal reproduction pointing to a loss of dispersal capacity

411 (Patiño et al., 2013a), range size did not correlate with any dispersal life-history trait. A

412 significant phylogenetic signal was found in variation of elevation range in

413 Amentuloradula, and in variation of biome range in Volutoradula and Metaradula,

414 whereas no such signal was detected within the large and widespread subgenus Radula.

415 Although the usefulness of metrics such as Pagel's $\lambda$ in diagnosing phylogenetic niche

416 conservatism has been questioned (for review see Crisp and Cook, 2012), it is tempting to

417 interpret this pattern in terms of phylogenetic niche conservatism. A thorough

418 investigation of the evolution of ecological niches, employing actual environmental 
419 measurements, would, however, be necessary to determine whether niche conservatism

420 indeed constrains the ability of certain lineages to enlarge their geographic distribution

421 ranges.

422 Despite their greatly restricted distribution to Australasia and the Neotropics,

423 Amentuloradula and Volutoradula did not exhibit significantly lower diversification rates

424 than more widespread lineages, in contrast with Rosenzweig (1995)'s prediction that the

425 probability of speciation increases with range size by promoting geographic isolation and

426 increasing the rate at which novel habitats are encountered. Indeed, the

427 macroevolutionary cohort analysis implemented in BAMM suggested that subgenera

428 Radula and Cladoradula, which exhibit a nearly cosmopolitan distribution, have a

429 different set of macroevolutionary rate parameters with respect to the rest of clades, but

430 also between them. In addition, subgenus Radula had higher net diversification rates than

431 the clades restricted to the Neotropics and Australasia, but this pattern was not confirmed

432 in Cladoradula. Rosindell and Phillimore (2011) suggested that, in highly mobile

433 organisms, speciation rates depend on the trade-off between the number of events

434 potentially fostering anagenesis and the intensity of migration preventing speciation

435 through undisrupted gene flow. In this context, one potential explanation to the equivocal

436 pattern here observed is that stochastic long-distance dispersal events are likely to

437 balance allele frequencies across large spatial scales, leading to low genetic structure

438 among geographically distant areas or even continents (Cronberg et al., 1997; McDaniel

439 and Shaw, 2003; Pisa et al., 2014; Shaw et al., 2015), ultimately decreasing the speciation

440 rates in highly mobile, widespread lineages. 


\section{Acknowledgements}

443 The Belgian Fund for Scientific Research (FNRS), the Leopold III Funds, and University

444 of Liege, and the European Union's Seventh Framework Programme provided funding

445 for this research. J.P. also acknowledges funding by Spain's Ministerio de Ciencia e

446 Innovación (Juan de la Cierva Program - Incorporation). The work was also supported by

447 the U.S. National Science Foundation (grant number, EF-0531730-002 to AJS). We

448 thank three anonymous referees for constructive remarks.

450 References

451 Abellán, P., Ribera, I., 2011. Geographic location and phylogeny are the main determinants of the size of the geographical range in aquatic beetles. BMC Evol. Biol. 11, 344.

454 Aigoin, D.A., Devos, N., Huttunen, S., Ignatov, M.S., Gonzalez-Mancebo, J.M.,

455 Vanderpoorten, A., 2009. And if Engler was not completely wrong? Evidence for multiple evolutionary origins in the moss flora of Macaronesia. Evolution $63,3248-3257$.

458 Baele, G., Lemey, P., Bedford, T., Rambaut, A., Suchard, M.A., Alekseyenko, A.V., 2012. Improving the accuracy of demographic and molecular clock model comparison while accommodating phylogenetic uncertainty. Mol. Biol. Evol.

462 Blomberg, S.P., Garland, T., Ives, A.R., 2003. Testing for phylogenetic signal in 463 comparative data: behavioral traits are more labile. Evolution 57, 717-745.

464 Borregaard, M.K., Gotelli, N.J., Rahbek, C., 2012. Are range-size distributions 465 consistent with species-level heritability? Evolution 66, 2216-2226. 
466 Chacón, J., Renner, S.S., 2014. Assessing model sensitivity in ancestral area reconstruction using Lagrange: a case study using the Colchicaceae family. J. Biogeogr. 41, 1414-1427.

469 Cooper, E.D., Henwood, M.J., Brown, E.A., 2012. Are the liverworts really that old?

$470 \quad$ Cretaceous origins and Cenozoic diversifications in Lepidoziaceae reflect a recurrent theme in liverwort evolution. Biol. J. Linnean Soc. 107, 425-441.

472 Crisp, M.D., Cook, L.G., 2012. Phylogenetic niche conservatism: what are the underlying evolutionary and ecological causes? New Phytol. 196, 681-694.

474 Cronberg, N., Molau, U., Sonesson, M., 1997. Genetic variation in the clonal bryophyte Hylocomium splendens at hierarchical geographical scales in Scandinavia. Heredity 78, 293-301.

Devos, N., Renner, M.A.M., Gradstein, R., Shaw, A.J., Laenen, B., Vanderpoorten, A., 2011a. Evolution of sexual systems, dispersal strategies and habitat selection in the liverwort genus Radula. New Phytol. 192, 225-236.

Cusimano, N., Renner, S.S., 2014. Ultrametric trees or phylograms for ancestral state reconstruction: does it matter. Taxon 63, 721-726.

Devos, N., Renner, M.A.M., Gradstein, S.R., Shaw, J., Vanderpoorten, A., 2011 b. Molecular data challenge traditional subgeneric divisions in the leafy liverwort Radula. Taxon 60, 1623-1632.

Drummond, A.J., Suchard, M.A., Xie, D., Rambaut, A., 2012. Bayesian phylogenetics with BEAUti and the BEAST 1.7. Mol. Biol. Evol. 29, 1969-1973.

Ericson, P.G.P., Christidis, L., Cooper, A., Irestedt, M., Jackson, J., Johansson, U.S., Norman, J.A., 2002. A Gondwanan origin of passerine birds supported by DNA sequences of the endemic New Zealand wrens. Proc. R. Soc. B 269, 235-241.

Feldberg, K., Schneider, H., Stadler, T., Schäfer-Verwimp, A., Schmidt, A.R., Heinrichs, J., 2014. Epiphytic leafy liverworts diversified in angiospermdominated forests. Sci. Rep. 4, 5974.

493 Fine, P.V.A., 2015. Ecological and evolutionary drivers of geographic variation in 494 species diversity. Annu. Rev. Ecol. Evol. Syst. 46, 369-392. 
495 Gaston, K.J., 1996. Species-range-size distributions: patterns, mechanisms and implications. Trends Ecol. Evol. 11, 197-201.

Gaston, K.J., 1998. Species-range size distributions: products of speciation, extinction and transformation. Phil. Trans. R. Soc. B. 353, 219-230.

Goldberg, E.E., Lancaster, T.L., Ree, R.H., 2011. Phylogenetic inference of reciprocal effects between geographic range evolution and diversification. Syst. Biol. 60, 451-465.

Hedges, S.B., Parker, P.H., Sibley, C.G., Kumar, S.S., 1996. Continental breakup and the ordinal diversification of birds and mammals. Nature 381, 226-228.

Hunt, G., Roy, K., Jablonski, D., 2005. Species- level heritability reaffirmed: A comment on "on the heritability of geographic range sizes". Am. Nat. 166, 129135.

509 Jablonski, D., Roy, K. 2003. Geographical range and speciation in fossil and living

510 molluscs. Proc. R. Soc. Lond. B (2003) 270, 401-406.

511 Jones, K.E., Sechrest, W., Gittleman, J.L., 2005. Age and area revisited: identifying 512 global patterns and implications for conservation. Phylogeny and conservation.

513 Cambridge University Press, Cambridge, 141-165.

514 Kisel, Y., Barraclough, T.G., 2010. Speciation has a spatial scale that depends on levels 515 of gene flow. Am. Nat. 175, 316-334.

516 Korall, P., Pryer, K.M., 2014. Global biogeography of scaly tree ferns (Cyatheaceae): evidence for Gondwanan vicariance and limited transoceanic dispersal. J.

518 Biogeogr. 41, 402-413.

519 Laenen, B., Shaw, B., Schneider, H., Goffinet, B., Paradis, E., Désamoré, A., Heinrichs, 520 J., Villarreal, J.C., Gradstein, S.R., McDaniel, S.F., Long, D.G., Forrest, L.L., 521 Hollingsworth, M.L., Crandall-Stotler, B., Davis, E.C., Engel, J., Von Konrat,

522 M., Cooper, E.D., Patiño, J., Cox, C.J., Vanderpoorten, A., Shaw, A.J., 2014.

523 Extant diversity of bryophytes emerged from successive post-Mesozoic

524 diversification bursts. Nat. Comm. 5, 5134. 
525 Laenen, B., Machac, A., Gradstein, S.R., Shaw, B., Patiño, J., Désamoré, A., Goffinet,

526 B., Cox, C.J., Shaw, J., Vanderpoorten, A., 2016. Geographical range in

527 liverworts: does sex really matter? J. Biogeogr. 43, 627-635.

528 Lambert, S.M., Wiens, J.J., 2013. Evolution of viviparity: A phylogenetic test of the

529 cold-climate hypothesis in phrynosomatid lizards. Evolution 67, 2614-2630.

530 Laube, I., Graham, C.H., Böhning-Gaese, K., 2013a. Intra-generic species richness and

531 dispersal ability interact to determine geographic ranges of birds. Global Ecol.

532 Biogeogr. 22, 223-232.

533 Laube, I., Korntheuer, H., Schwager, M., Trautmann, S., Rahbek, C., Böhning-Gaese,

534 K., 2013b. Towards a more mechanistic understanding of traits and range sizes.

$535 \quad$ Global Ecol. Biogeogr. 22, 233-241.

536 Lester, S.E., Ruttenberg, B.I., Gaines, S.D., Kinlan, B.P., 2007. The relationship

537 between dispersal ability and geographic range size. Ecol. Lett. 10, 745-758.

538 Lewis, L.R., Rozzi, R., Goffinet, B., 2014. Direct long-distance dispersal shapes a New

539 World amphitropical disjunction in the dispersal-limited dung moss Tetraplodon

540 (Bryopsida: Splachnaceae). J. Biogeogr. 41, 2385-2395.

541 Losos, J.B., Alder, F.R., 1996. Stumped by trees - a generalized null model for patterns 542 of organismal diversity. Am. Nat. 145, 329-342.

543 Machac, A., Zrzavý, J., Storch, D., 2011. Range size heritability in carnivora is driven

$544 \quad$ by geographic constraints. Am. Nat. 177, 767-779.

545 Mao, K., Milne, R.I., Zhang, L., Peng, Y., Liu, J., Thomas, P., Mill, R.R., Renner, S.S., 546 2012. Distribution of living Cupressaceae reflects the breakup of Pangea. Proc.

$547 \quad$ Natl. Acad. Sci. U.S.A. 109, 7793-7798.

548 Matzke, N.J., 2013. Probabilistic historical biogeography: new models for founder-

549 event speciation, imperfect detection, and fossils allow improved accuracy and

550 model-testing. Front. Biogeogr. 5, 242-248.

551 Matzke, N.J., 2014. Model selection in historical biogeography reveals that founder-

552 event speciation is a crucial process in island clades. Syst. Biol. 63, 951-970.

553 McDaniel, S.F., Shaw, A.J., 2003. Phylogeographic structure and cryptic speciation in

554 the trans-antarctic moss Pyrrhobryum mnioides. Evolution 57, 205-215. 
555 Mooers, A.Ø., Schluter, D., 1999. Reconstructing ancestor states with maximum likelihood: support for one-and two-rate models. Syst. Biol. 48, 623-633.

557 Münkemüller, T., Lavergne, S., Bzeznik, B., Dray, S., Jombart, T., Schiffers, K., Thuiller, W., 2012. How to measure and test phylogenetic signal. Methods Ecol.

560 Nylander, J.A.A., 2004. MrModeltest v2. Evolutionary Biology Centre, Uppsala $561 \quad$ University, Uppsala, Sweden.

562 Olson, D.M., Dinerstein, E., Wikramanayake, E.D., Burgess, N.D., Powell, G.V.N., 563 Underwood, E.C., D'Amico, J.A., Itoua, I., Strand, H.E., Morrison, J.C., Loucks, C.J., Allnutt, T.F., Ricketts, T.H., Kura, Y., Lamoreux, J.F., Wettengel, W.W., Hedao, P., Kassem, K.R., 2001. Terrestrial ecoregions of the world: A new map of life on earth. BioScience 51, 933-938.

567 Pagel, M., 1999a. Inferring the historical patterns of biological evolution. Nature 401, $568 \quad 877-884$.

569 Pagel, M., 1999b. The maximum likelihood approach to reconstructing ancestral character states of discrete characters on phylogenies. Syst. Biol. 48, 612-622.

571 Pagel, M., Meade, A., Barker, D., 2004. Bayesian estimation of ancestral character 572 states on phylogenies. Syst. Biol. 53, 673-684.

573 Patiño, J., Bisang, I., Hedenäs, L., Dirkse, G., Bjarnason, Á.H., Ah-Peng, C.,

$574 \quad$ Vanderpoorten, A., 2013a. Baker's law and the island syndromes in bryophytes.

$575 \quad$ J. Ecol. 101, 1245-1255.

576 Patiño, J., Devos, N., Vanderpoorten, A., Schäfer-Verwimp, A., Renner, M., 2013b.

577 The identity of Radula carringtonii Jack. J. Bryol. 35, 314-316.

578 Patiño, J., Goffinet, B., Sim-Sim, M., Vanderpoorten, A., 2016. Is the sword moss

579 (Bryoxiphium) a preglacial Tertiary relict? Mol. Phylogenet. Evol. 96, 200-206.

580 Pellicer, J., Kelly, L.J., Leitch, I.J., Zomlefer, W.B., Fay, M.F., 2014. A universe of

581 dwarfs and giants: genome size and chromosome evolution in the monocot

582 family Melanthiaceae. New Phytol. 201, 1484-1497. 
583 Pirie, M.D., Humphreys, A.M., Antonelli, A., Galley, C., Linder, H.P., 2012. Model

584 uncertainty in ancestral area reconstruction: A parsimonious solution? Taxon 61, $585 \quad 652-664$.

586 Pisa, S., Biersma, E.M., Convey, P., Patiño, J., Vanderpoorten, A., Werner, O., Ros, 587 R.M., 2014. The cosmopolitan moss Bryum argenteum in Antarctica: recent $588 \quad$ colonisation or in situ survival? Polar Biol. 37, 1469-1477.

589 Pyron, R.A., 2014. Biogeographic analysis reveals ancient continental vicariance and 590 recent oceanic dispersal in amphibians. Syst. Biol. 63, 779-797.

591 Quental, T.B., Marshall, C.R., 2009. Extinction during evolutionary radiations: Reconciling the fossil record with molecular phylogenies. Evolution 63, 31583167.

Quental, T.B., Marshall, C.R., 2010. Diversity dynamics: molecular phylogenies need the fossil record. Trends Ecol. Evol. 25, 434-441.

Rabosky, D.L., 2014. Automatic detection of key innovations, rate shifts, and diversitydependence on phylogenetic trees. PLoS ONE 9, e89543. visualization of complex macroevolutionary dynamics: An example from australian scincid lizards. Syst. Biol. 63, 610-627. J.G., 2014b. BAMMtools: an R package for the analysis of evolutionary dynamics on phylogenetic trees. Methods Ecol. Evol. 5, 701-707. ability explain differences in range size among sister-taxa pairs of Collinsia (Plantaginaceae)? An extension of Baker's Law. New Phytol. 183, 618-629. 
613 Renner, M.A.M., Devos, N., Brown, E.A., von Konrat, M.J., 2014. A revision of 614 Australian species of Radula subg. Odontoradula. Austral. Syst. Bot. 26, 408$615 \quad 447$.

616 Renner, M.A.M., Devos, N., Patino, J., Brown, E., Orme, A., Elgy, M., Wilson, T., 617 Gray, L., von Konrat, M., 2013. Integrative taxonomy resolves the cryptic and 618 pseudo-cryptic Radula buccinifera complex (Porellales, Jungermanniopsida), 619 including two reinstated and five new species. PhytoKeys 27, 1-113.

620 Revell, L.J., 2012. Phytools: an R package for phylogenetic comparative biology (and 621 other things). Methods Ecol. Evol. 3, 217-223.

624 Rosindell, J., Phillimore, A.B., 2011. A unified model of island biogeography sheds 625 light on the zone of radiation. Ecol. Lett. 14, 552-560.

626 Sanmartín, I., Ronquist, F., 2004. Southern hemisphere biogeography inferred by event627 based models: Plant versus animal patterns. Syst. Biol. 53, 216-243.

628 Sauquet, H., Ho, S.Y.W., Gandolfo, M.A., Jordan, G.J., Wilf, P., Cantrill, D.J., Bayly, 629 M.J., Bromham, L., Brown, G.K., Carpenter, R.J., Lee, D.M., Murphy, D.J., Sniderman, J.M.K., Udovicic, F., 2012. Testing the impact of calibration on molecular divergence times using a fossil-rich group: the case of Nothofagus (Fagales). Syst. Biol. 61, 289-313.

Shaw, A.J., Shaw, B., Johnson, M.G., Devos, N., Stenøien, H.K., Flatberg, K.I., Carter, B.E., 2015. Phylogenetic structure and biogeography of the Pacific Rim clade of Sphagnum subgen. Subsecunda: haploid and allodiploid taxa. Biol. J. Linnean Soc. 116, 295-311.

Sheth, S.N., Jiménez, I., Angert, A.L., 2014. Identifying the paths leading to variation in geographical range size in western North American monkeyflowers. J. Biogeogr. 41, 2344-2356.

Sun, Y., He, X., Glenny, D., 2014. Transantarctic disjunctions in Schistochilaceae (Marchantiophyta) explained by early extinction events, post-Gondwanan radiations and palaeoclimatic changes. Mol. Phylogenet. Evol. 76, 189-201. 
643 Swofford, D.L., 2002. PAUP*: Phylogenetic analysis using parsimony (*and other methods), version 4. Sinauer, Sunderland, MA.

645 Tan, B.C., Pócs, T., 2000. Bryogeography and conservation of bryophytes. In: Shaw, A.J., Goffinet, B. (Eds.), Bryophyte biology. Cambridge University Press, New York, pp. 403-448.

van der Wijk, R., Margadant, W.D., Florschütz, P.A., 1959. Index muscorum. International Association of Plant Taxonomists, Utrech.

Vanderpoorten, A., Gradstein, S.R., Carine, M.A., Devos, N., 2010. The ghosts of Gondwana and Laurasia in modern liverwort distributions. Biol. Rev. 85, 471487.

653 Vanderpoorten, A., Laenen, B., Rumsey, F., González-Mancebo, J., Gabriel, R., Carine, M., 2011. Dispersal, diversity and evolution of the Macaronesian cryptogamic floras. In: Bramwell, D., Caujapé-Castellls, J. (Eds.), The biology of island floras. Cambridge University Press, Cambridge, pp. 383-364. Erwin, D.H., Angstey, R.L. (Eds.), New approaches to speciation in the fossil record. Columbia University Press, New York, pp. 87-122.

Waldron, A., 2007. Null models of geographic range size evolution reaffirm its heritability. Am. Nat. 170, 221-231.

Webb, T.J., Gaston, K.J. 2003. On the heritability of geographic range sizes. Am. Nat. $161,553-566$.

Wessel, A., Hoch, H., Asche, M., von Rintelen, T., Stelbrink, B., Heck, V., Stone, F.D., Howarth, F.G., 2013. Founder effects initiated rapid species radiation in Hawaiian cave planthoppers. Proc. Natl. Acad. Sci. U.S.A. 110, 9391-9396.

Wilf, P., Escapa, I.H., 2015. Molecular dates require geologic testing. New Phytol. 209, $1359-1362$.

Wolf, P.G., Schneider, H., Ranker, T.A., 2001. Geographic distributions of homosporous ferns: does dispersal obscure evidence of vicariance? J. Biogeogr. $28,263-270$. 
672 Woodburne, M.O., Case, J.A., 1996. Dispersal, vicariance, and the Late Cretaceous to 673 early Tertiary land mammal biogeography from South America to Australia. J. 674 Mammal. Evol. 3, 121-161.

675 Wright, S., 1934. An analysis of variability in number of digits in an inbred strain of $676 \quad$ Guinea pigs. Genetics 19, 506-536.

677 Yamada, K., 2003. Radulaceae. In: Gradstein, S.R., Costa, D.P. (Eds.), The Hepaticae 678 and Anthocerotae of Brazil. Memoirs of the New York Botanical Garden 87. 679 New York Botanical Garden Press, New York.

680 Yu, Y., Harris, A.J., Blair, C., He, X., 2015. RASP (Reconstruct Ancestral State in 681 Phylogenies): A tool for historical biogeography. Mol. Phylogenet. Evol. 87, 46682 49.

683 


\section{Table 1}

685 Mean node age estimates (Ma) and 95\% highest posterior density (HPD) distribution of ages for Radula subgenera (see Fig. 1

686 for the location of each node). The posterior probabilities $(p p) \geq 0.95$ in BEAST and the ancestral areas inferred with a relative

687 probability $\geq 0.25$ with the best-fit model $(\mathrm{DEC}+\mathrm{J})$ in BIOGEOBEARS are also shown. An asterisk (*) indicates clades

688 predominantly Australasian, and a hash (\#) for mainly Neotropical ones (see Fig. 3). ? = not resolved. Area codes: E = Europe; F

$689=$ Africa $; \mathrm{S}=$ Asia; $\mathrm{N}=$ North America; $\mathrm{A}=$ Central and South America; $\mathrm{Z}=$ New Zealand; $\mathrm{T}=$ Australia; $\mathrm{O}=\mathrm{Oceania}$.

\begin{tabular}{|c|c|c|c|}
\hline Node (number) & Age (95\% HPD) & $p p$ & $\begin{array}{l}\text { [Ancestral range] } \\
\text { Relative Probability }\end{array}$ \\
\hline Subgenus Cladoradula split (109) & $144.6(109.6-197.5)$ & 1.00 & [?] \\
\hline Crown of subgenus Cladoradula (211) & $11.6(4.9-21.9)$ & 1.00 & [EFSA] 0.78 \\
\hline Subgenus Dactyloradula split (110) & $126.1(96.6-166.0)$ & 0.99 & [?] \\
\hline Subgenus Odontoradula split (111) & $101.2(78.5-129.2)$ & 1.00 & [?] \\
\hline Crown of subgenus Odontoradula (112) & $68.9(50.9-91.1)$ & 1.00 & [ATZ] 0.38 \\
\hline Split between subgenera Metaradula and Volutoradula (125) & $64.1(46.9-84.6)$ & 1.00 & [?] \\
\hline Crown of subgenus Volutoradula\# (126) & $38.3(25.7-54.1)$ & 1.00 & [A] 0.81 \\
\hline Crown of subgenus Metaradula* $(150)$ & $49.4(35.2-66.2)$ & 1.00 & [TZ] 0.34 \\
\hline Split between subgenera Radula and Amentuloradula (167) & $82.3(61.6-107.3)$ & 0.99 & [T] 0.18 \\
\hline Crown of subgenus Amentuloradula* (168) & $48.9(35.3-65.5)$ & 1.00 & [TZ] 0.80 \\
\hline Crown of subgenus Radula (184) & $45.5(30.8-64.5)$ & 1.00 & [NZ] 0.25 \\
\hline
\end{tabular}

690

691 
Table 2

693 Phylogenetic signal in continuous characters in the liverwort genus Radula as evaluated by 694 Blomberg's $K$ and Pagel's $\lambda . \%$ P, percentage of significant tests; (*), in Amentuloradula, 695 all the nodes immediately after the node 150 showed a strong phylogenetic signal for 696 elevation range by using Pagel's $\lambda$ (see Table S6).

\begin{tabular}{|c|c|c|c|c|c|c|}
\hline & \multicolumn{3}{|c|}{ Blomberg's $K$} & \multicolumn{3}{|c|}{ Pagel's lambda $\lambda$} \\
\hline & Mean & Max.-Min. & $\% P$ & Mean & Max.-Min. & $\% P$ \\
\hline \multicolumn{7}{|l|}{ Geographic range } \\
\hline Odontoradula (112) & 0.61 & $0.30-0.84$ & 0 & 0 & 0 & 0 \\
\hline Volutoradula (node 126) & 0.33 & $0.08-0.54$ & 64 & 0.57 & $0.48-0.84$ & 100 \\
\hline Metaradula (node 150) & 0.76 & $0.50-1.11$ & 3.4 & 0.56 & $0.07-1$ & 51.7 \\
\hline Amentuloradula (node 168) & 1.66 & $1.02-2.25$ & 100 & 0.98 & $0.79-1$ & 100 \\
\hline Radula (node 184) & 0.08 & $0.20-0.01$ & 0 & 0 & $>0.001-0.04$ & 0 \\
\hline \multicolumn{7}{|l|}{ Elevation range } \\
\hline Odontoradula (112) & 0.56 & $0.19-0.84$ & 0 & 0.01 & $>0.001-1$ & 1 \\
\hline Volutoradula (node 126) & 0.23 & $0.11-0.35$ & 0 & $>0.001$ & $>0.001$ & 0 \\
\hline Metaradula (node 150) & 0.61 & $0.45-0.85$ & 0 & 0.12 & $>0.001-0.83$ & 0 \\
\hline Amentuloradula (node 168) & 0.86 & $0.59-1.22$ & 19.48 & 0.79 & $>0.001-1$ & $79(*)$ \\
\hline Radula (node 184) & 0.08 & $0.02-0.19$ & 0 & $>0.001$ & $>0.001$ & 0 \\
\hline \multicolumn{7}{|l|}{ Biome range } \\
\hline Odontoradula (112) & 0.87 & $0.56-1.08$ & 1 & 0.85 & $>0.001-1$ & 69 \\
\hline Volutoradula (node 126) & 0.70 & $0.37-1$ & 88 & 0.93 & $>0.001-1$ & 61 \\
\hline Metaradula (node 150) & 0.87 & $0.53-1.25$ & 55 & 0.73 & $0.3-1$ & 33 \\
\hline Amentuloradula (node 168) & 0.46 & $0.23-0.73$ & 0 & $>0.001$ & $>0.001-0.2$ & 0 \\
\hline Radula (node 184) & 0.19 & $0.05-0.35$ & 0 & 0.31 & $0.16-0.43$ & 0 \\
\hline \multicolumn{7}{|l|}{ Substrate range } \\
\hline Odontoradula (112) & 0.34 & $0.11-0.69$ & 0 & 0.03 & $>0.001-1.58$ & 0 \\
\hline Volutoradula (node 126) & 0.26 & $0.09-0.46$ & 0 & $>0.001$ & $>0.001-0.39$ & 0 \\
\hline Metaradula (node 150) & 0.59 & $0.29-0.84$ & 15 & 0.60 & $0.27-0.78$ & 0 \\
\hline Amentuloradula (node 168) & 0.51 & $0.19-1.00$ & 0 & 0.04 & $>0.001-0.51$ & 2.4 \\
\hline Radula (node 184) & 0.22 & $0.06-0.67$ & 2.9 & $>0.001$ & $>0.001$ & 0 \\
\hline
\end{tabular}


Table 3

699 Performance, as assessed by log-likelihood ( $\mathrm{lnL}$ ) and the Akaike information criterion

700 corrected for small sample sizes (AICc), of competing models of ancestral-area estimation

701 (dispersal-extinction-cladogenesis, DEC; dispersal-vicariance analysis, DIVA; BayArea),

702 as well as the same three models allowing for founder-event speciation $(+\mathrm{J})$ in the

703 liverwort genus Radula; n, number of parameters; $d$, rate of dispersal; $e$, rate of extinction;

$704 j$, relative probability of founder-event speciation. The best model is highlighted in bold.

705

\begin{tabular}{rrrrrrrr}
\hline & $\mathrm{Ln} L$ & AIC & AICc & $n$ & $d$ & $e$ & $j$ \\
\hline DEC & -336.872 & 677.744 & 677.858 & 2 & 0.0122 & 0.0052 & 0.0000 \\
DEC+J & $\mathbf{- 3 1 6 . 6 7 3}$ & $\mathbf{6 3 9 . 3 4 6}$ & $\mathbf{6 3 9 . 5 7 7}$ & $\mathbf{3}$ & $\mathbf{0 . 0 0 7 1}$ & $\mathbf{0 . 0 0 0 0}$ & $\mathbf{0 . 1 2 0 4}$ \\
DIVALIKE & -333.106 & 670.212 & 670.326 & 2 & 0.0128 & 0.0030 & 0.0000 \\
DIVALIKE+J & -320.588 & 647.176 & 647.407 & 3 & 0.0086 & 0.0000 & 0.0916 \\
BAYAREALIKE & -390.233 & 784.466 & 784.580 & 2 & 0.0157 & 0.0363 & 0.0000 \\
BAYAREALIKE+J & -327.235 & 660.470 & 660.701 & 3 & 0.0062 & 0.0037 & 0.1262 \\
\hline
\end{tabular}

706

707 
Fig. 1. Dated chronogram of the genus-level phylogenies of the genus Radula. Asterisks $(*)$ show the posterior probability from BEAST analyses $(\mathrm{pp}>0.95)$.

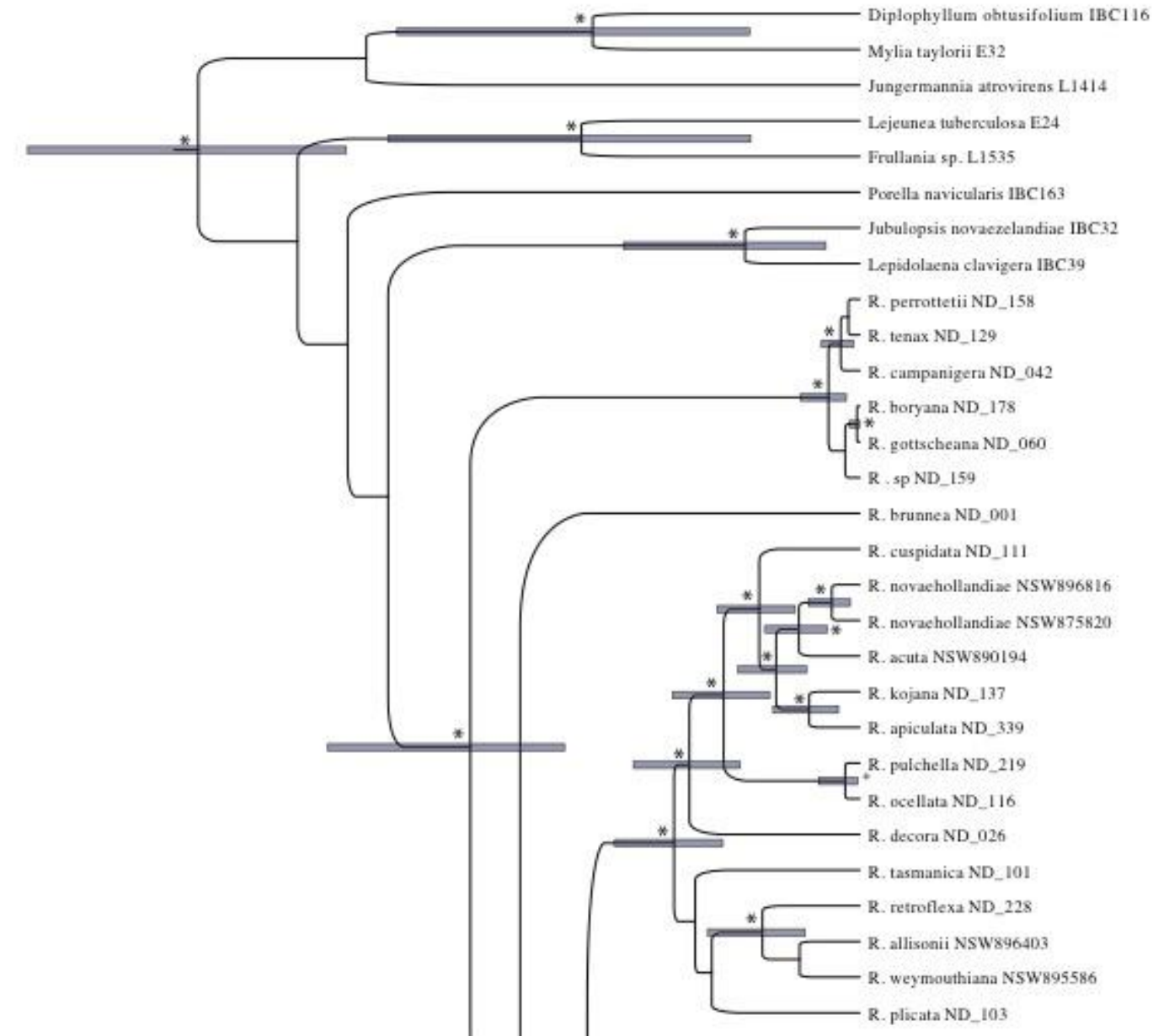


Fig. 1 (continued)

741

742

743

744

745

746

747

748

749

750

751

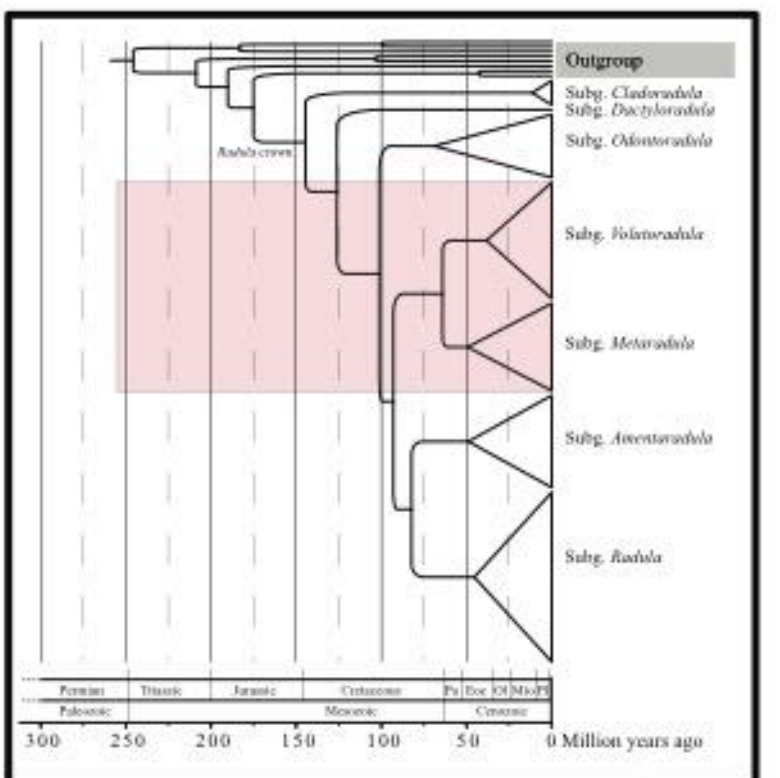

752

753

754

755

756

757

758

759

760

761

762

763

764

765

766

767

768

769
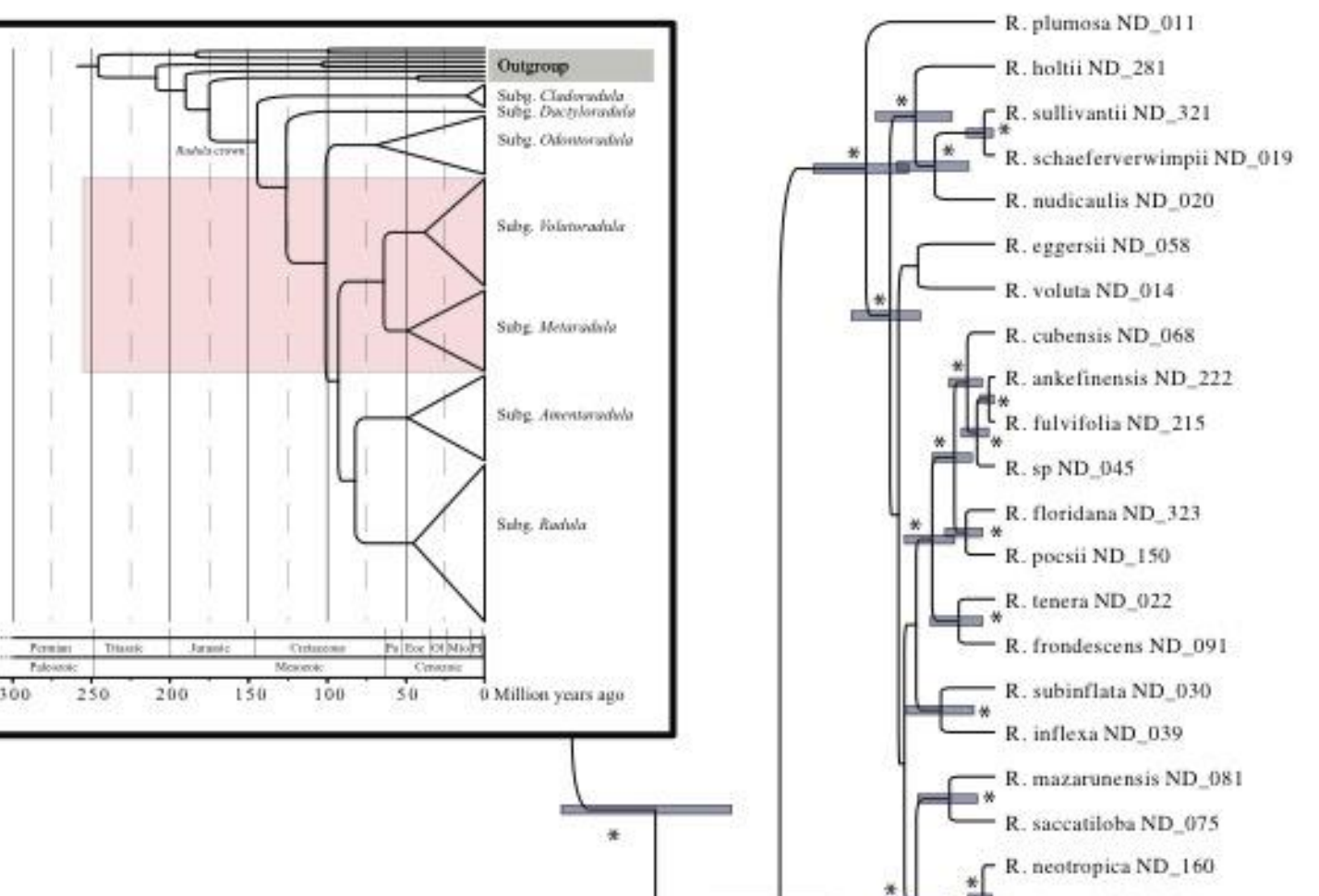

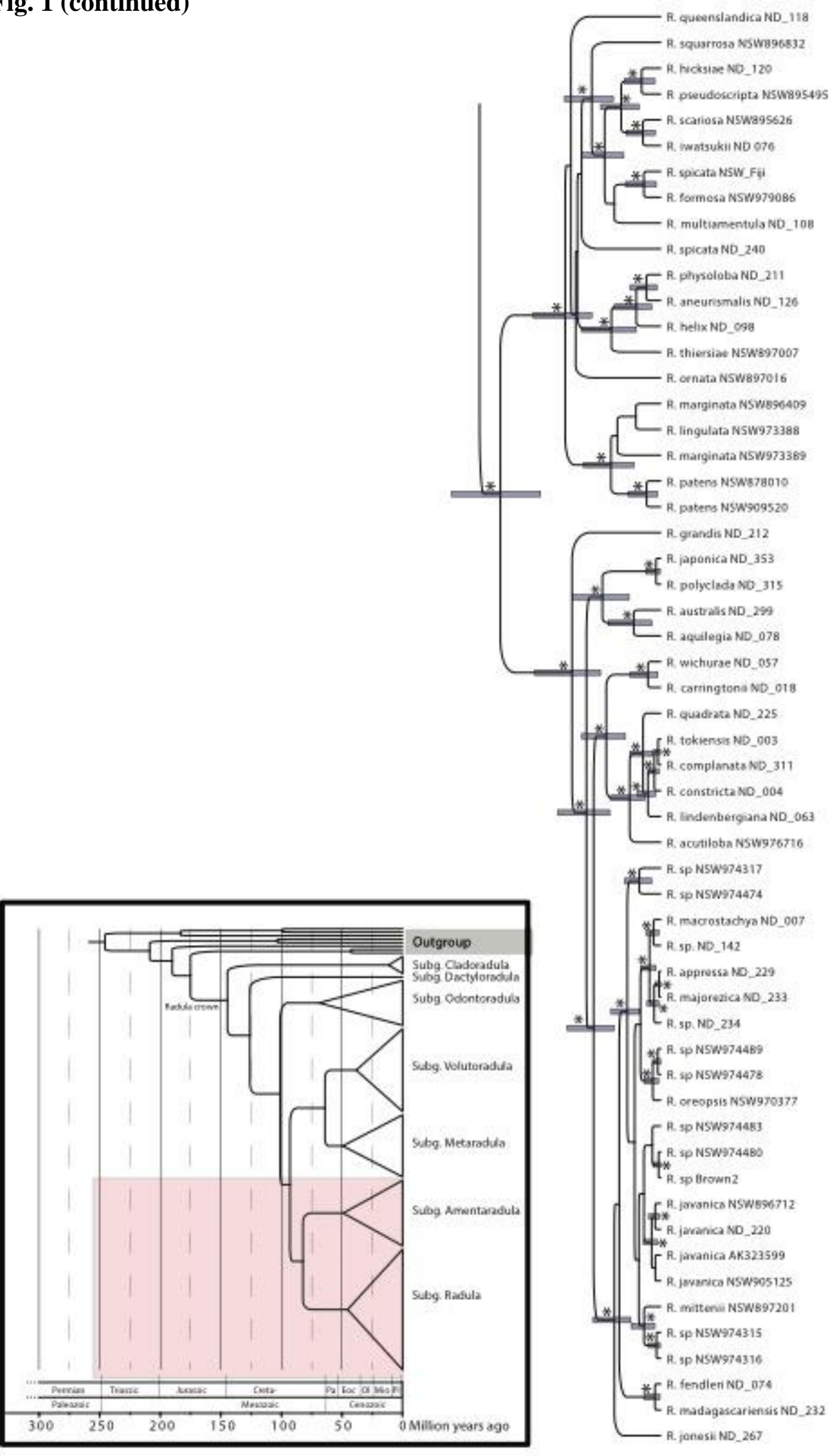
803 Fig. 2. Phylogenetic signal in geographic (A), elevation (B) and biome range (C) evaluated

804 by Pagel's lambda $(\lambda)$. Results using Blomberg's $K$ are shown in Fig. S1. Pie diagrams

805 represent the percentage of significant (green) and non-significant tests (grey) in the 100

806 replicates performed. The size of the pie diagrams indicates the value of $\lambda$. Bar plots

807 represent mean values \pm SD for each subgenus. Number of species here included are

808 shown in brackets.

809

810

811

812

813

814

A) Geographic range
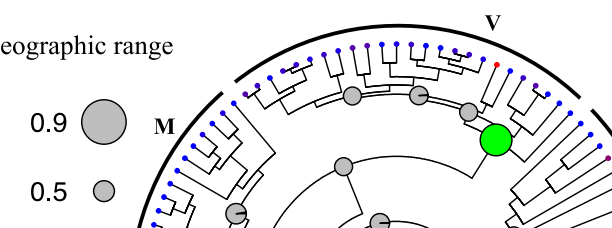

$0 \circ$
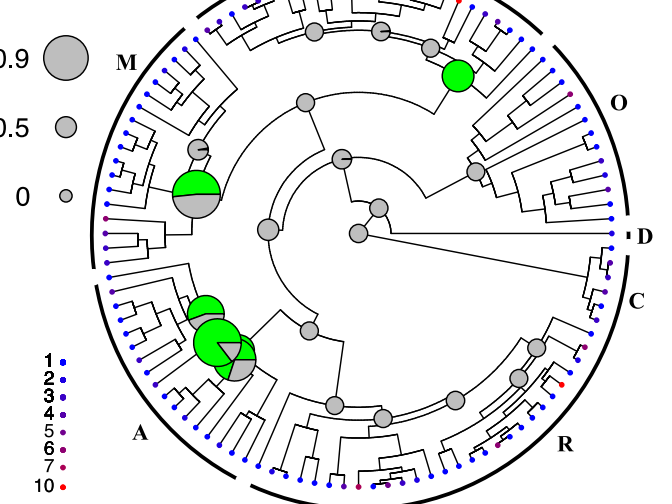

B) Elevation range

815

816

817

818

819

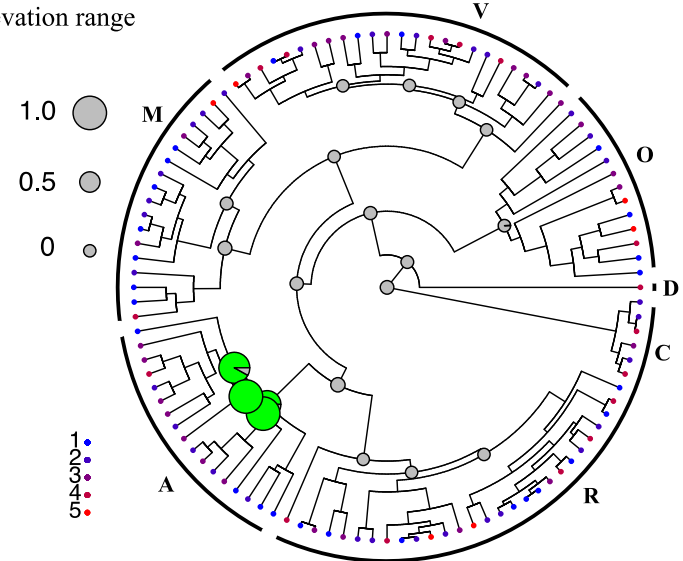

820

C) Biome range

821

822

823

824
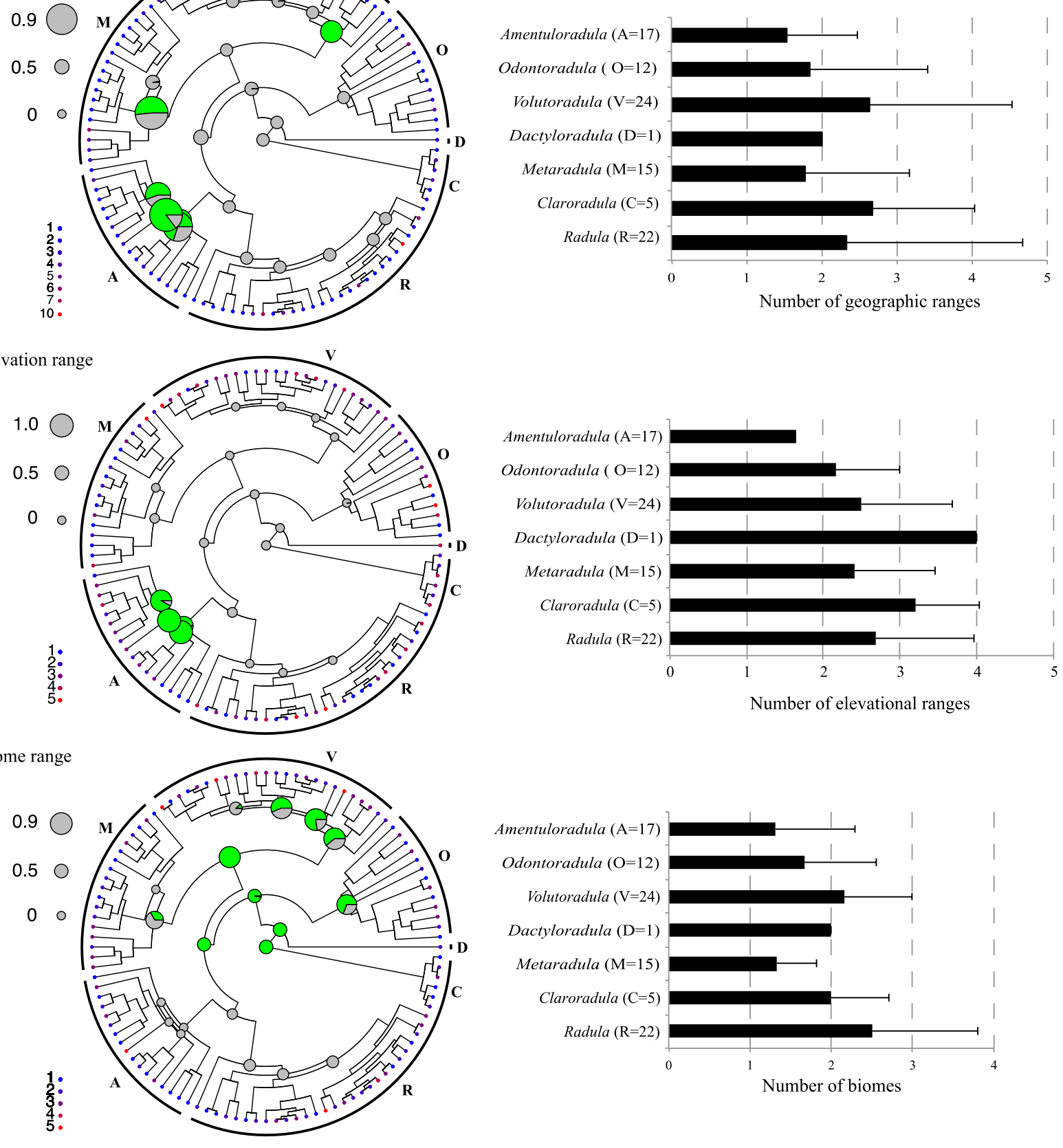
825 Fig. 3. Probabilities of ancestral area distributions under the DEC $+\mathrm{J}$ models in the genus Radula.

826 A, South America, light blue; N, North America, light green; Z, New Zealand, violet; T,

827 Australia, dark blue; O, Oceania, pink; E, Europe, red; F, Africa, yellow; S, Asia, green. The

828 corner positions represent geographical ranges immediately after a speciation event. The

829 maximum number of ancestral areas at a given node was five (parameters: $d=0.0071 ; e=0 ; j=$ 830 0.1204; $\mathrm{Ln} L=-316.99)$.

831

832

833

834

835

836

837

838

839

840

841

842

843

844

845

846

847

848

849

850

851

852

853

854

855

856

857

858

859

860

861

862

863

864

865

866

867

868

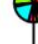

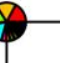

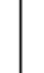


869 Fig. 4. Inferred net diversification rates $(\lambda-\mu ; \lambda=$ speciation and $\mu=$ extinction) among lineages and over time during the

870 evolution of the genus Radula. The phylogeny shows a time-calibrated maximum clade credibility tree, with branches colored

871 by reconstructed net diversification rates (a), which are means of the marginal densities of branch-specific rates. The location of

872 the maximum credibility rate shift configuration inferred by BAMM is also shown with a red circle indicative of a rate increase.

873 The circle size is proportional to the marginal probability of the shift (see also Fig. S2). Net-diversification-through-time plot for

874 several Radula clades (Table 1) are also included (b-g). Colour density shading illustrates the 95\% confidence interval of

875 estimated diversification rate at any point in time.
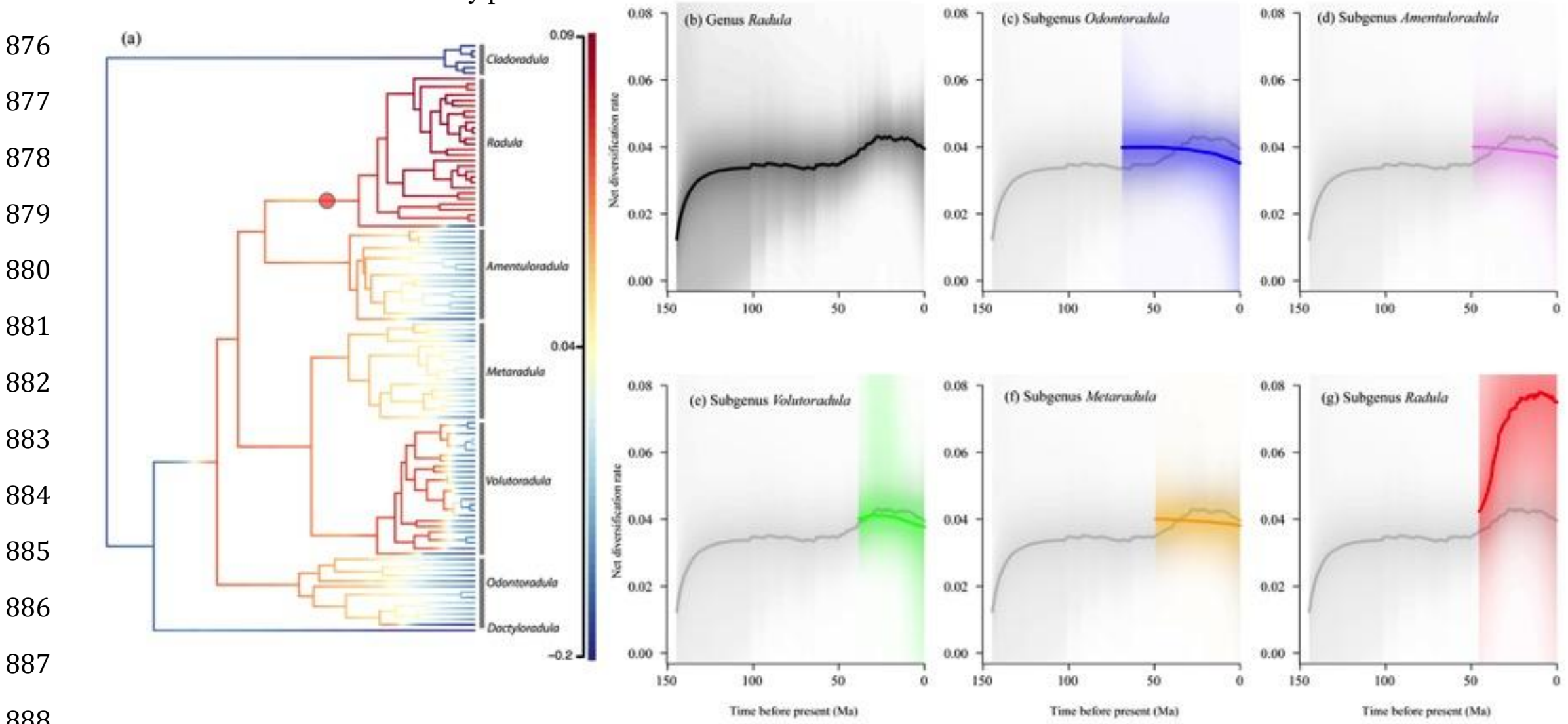
889 Fig. 5. Macroevolutionary cohort matrices for speciation in the liverwort genus Radula.

890 Each cell in the matrix is coded by a color denoting the pairwise probability that two

891 species share a common macroevolutionary rate regime. The maximum clade credibility

892 trees obtained in BEAST analyses is shown for reference on the left and upper margins of

893 each cohort matrix, but pairwise probabilities are averages from BAMM analyses

894 conducted over a sample of trees from the posterior distribution of topologies and branch

895 lengths. For speciation, it is evident that all species have highly correlated rate dynamics,

896 except for the subgenera Radula, Cladoradula and Dactyloradula.

897

898

899

900

901

902

903

904

905

906

907

908

909

910

911
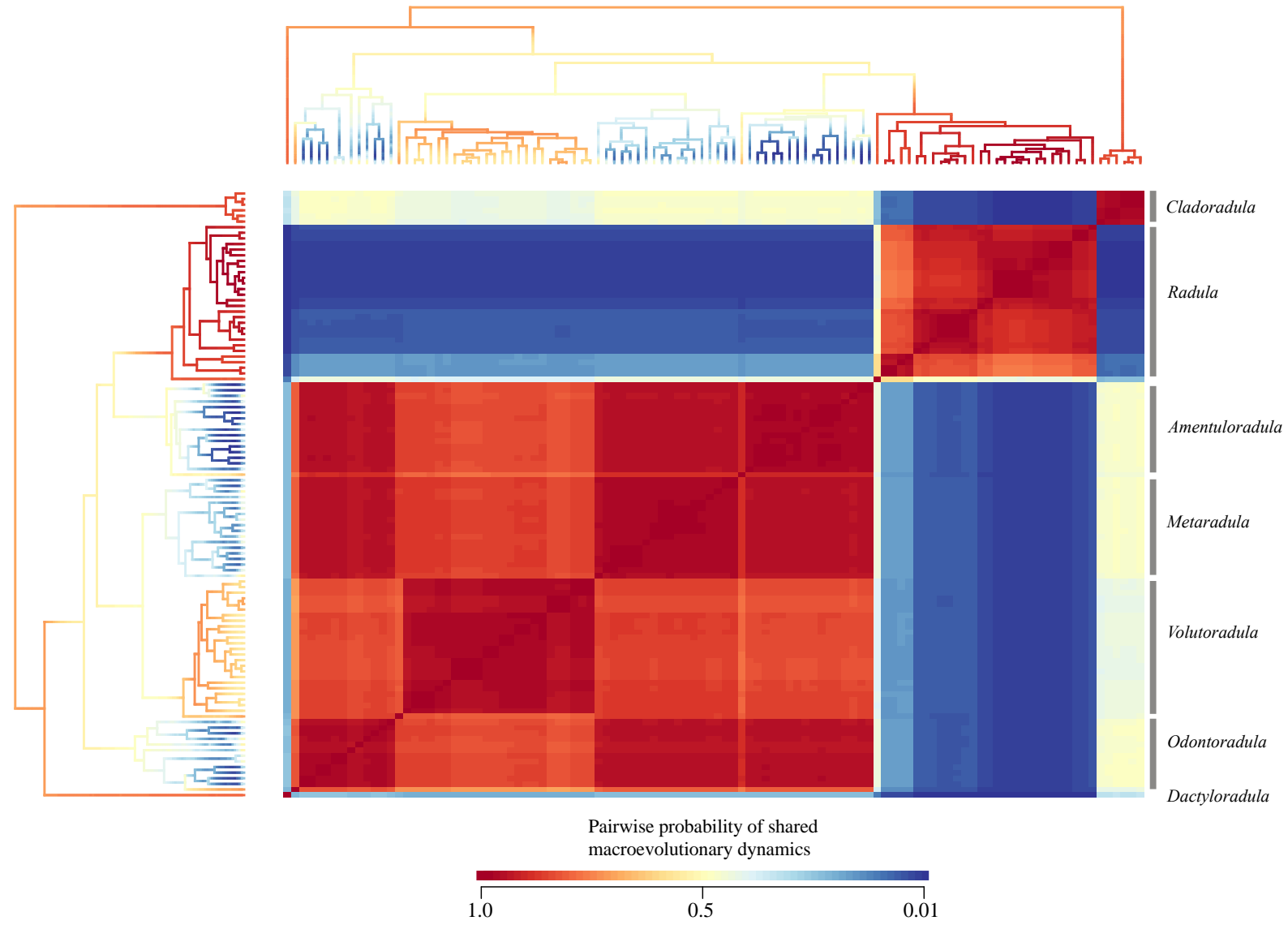

912

913

914 

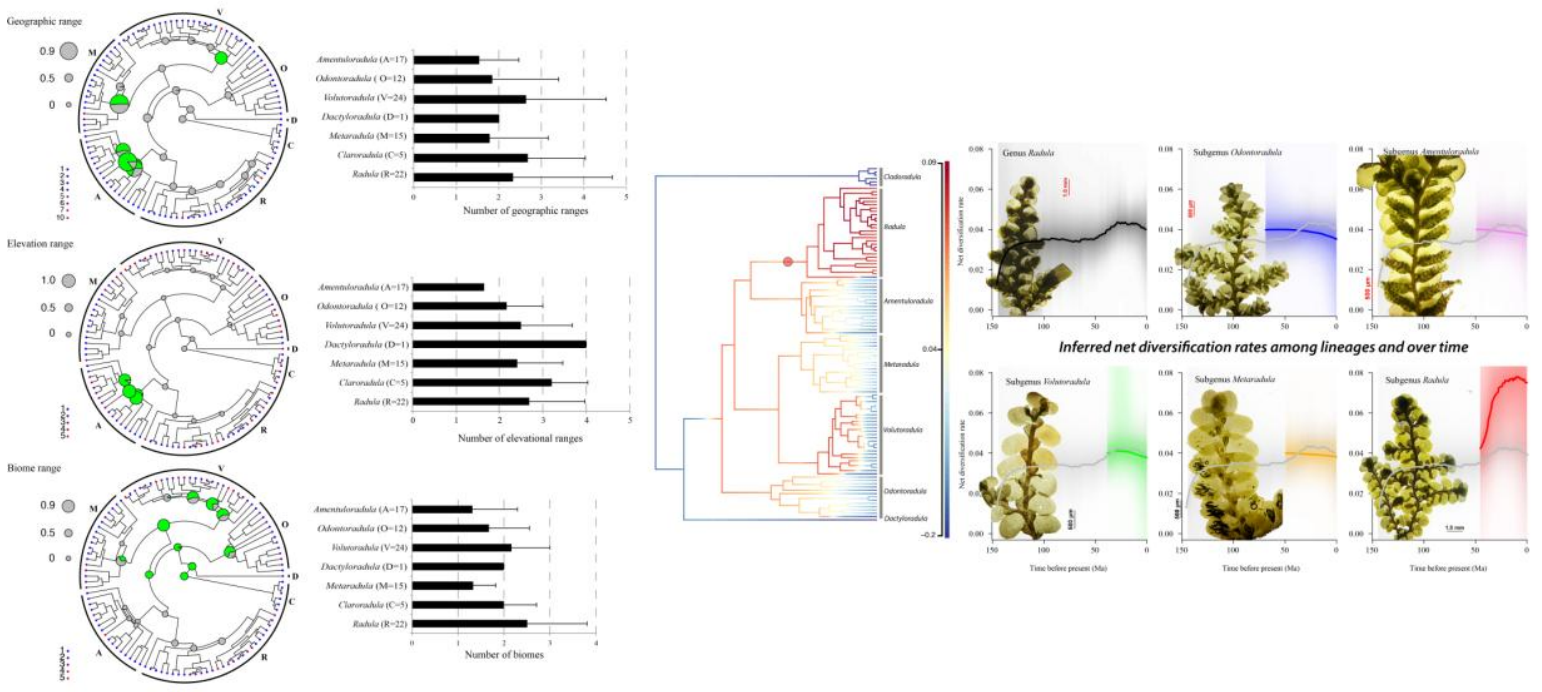

Phylogenetic signal evaluated by Pagel's lambda 